\title{
Investigations on the Doping Effect of Sodium Thiosulphate on KDP Single Crystals
}

\author{
Ramesh Rajendran \\ Department of Physics, S.T.Hindu college, Nagercoil- 629 002, India.
}

\begin{abstract}
:
Crystals of pure and sodium thiosulphate doped KDP were grown by slow evaporation technique. Sodium thiosulphate was used as a dopant, incorporated into the parent crystal (KDP) in $1 \mathrm{~mol} \%$ in order to improve the photoconductivity characteristics of KDP crystal. Optical absorption and photoconductivity properties were studied for pure and sodium thiosulphate doped KDP crystals. The pure and doped crystals were characterized by powder XRD analysis, primarily which was used to study the crystals structure and its perfection. Photoconductivity measurements confirmed the positive photoconductive nature of the doped crystal. DRSUV studies showed the crystals absorption percentage and its transparency. FTIR analysis confirmed the functional groups of the grown crystals.
\end{abstract}

Keywords: Crystal growth, photoconductivity studies, FTIR, DRS-UV.

\section{INTRODUCTION}

With current advances in molecular design and material engineering, inorganic materials are becoming highly attractive for photonic applications because of the possibility of tailoring their optical properties, physical properties and easier to synthesize. Traditionally the inorganic materials have been attracted interest in the recent past due to its various ability to be processed into crystals. Often these have high melting point, and high degree of chemical inertness. Hightemperature oxide materials are well studied for diverse applications. Potassium Dihydrogen Phosphate (KDP) crystal also known as Monopotassium phosphate(MKP) crystal is one such inorganic material. Several research works have been carried out on this material because of its very high laser damage.KDP has also been used in electro-optical modulator, $\mathrm{Q}$ switches, high power laser frequency conversion material, frequency doubling of dyer laser, shutter for high speed photography etc. In an attempt to improve its properties, many researchers have doped KDP crystals with different organic and inorganic substances.

Photoconductivity has been traditionally playing a significant role in materials research [1]. Photoactive materials and photoconductivity in materials play a large part in energy production, energy conservation, all kinds of detectors, catalysis and biological applications. Photovoltaics, photo detectors, and photo catalysis have been used for a long time to extract energy from photons. The study of photoconductivity is one of the most effective ways to investigate the properties of solids. It is a process which provides useful and valuable information about physical properties of materials and offer applications in photo resistors, photo detector, radiation measurement, it also has practical applications in television cameras, infrared detector, light meter and indirectly in photographic process. Other major applications in which photoconductivity plays a central role are normal silver halide emulsion photography and the very large field of electro photographic reproduction. Photoconductivity is one of an important phenomenon of solid state physics, involving the interaction of light quanta with a solid to produce free charge carriers whose subsequently reflects all of the fascinating diversions encountered by an electron or hole in a solid. Classic examples of photoconductivity materials include the conductive polymer polyvinylcarbazole used extensively in photocopying (Xerography), lead sulfide, used in infrared detection application, sodium thiosulphate used in photographic processing etc [2].

Sodium thiosulfate, $\mathrm{Na}_{2} \mathrm{~S}_{2} \mathrm{O}_{3}$, is a colorless crystalline inorganic compound that is more familiar as the pentahydrate, $\mathrm{Na}_{2} \mathrm{~S}_{2} \mathrm{O}_{3} \cdot 5 \mathrm{H}_{2} \mathrm{O}$. Sodium thiosulfate is hygroscopic and is sensitive to air and light. Because it dissolves silver salts, its major use is in photography for developing film where it fixes black and white negative, and prints after the developing stage. It is expected to be worth and interesting to dope KDP crystal with sodium thiosulphate for photography applications i.e. as photographic fixer, shutter for photography etc. Sodium thiosulfate is produced chiefly from liquid waste products of sodium sulfide or sulfur dye manufacture.

In this research work pure KDP crystal and sodium thiosulphate doped KDP crystal were grown by slow evaporation method. The grown crystals were subjected to various characteristics studies like Powder X-ray diffraction, photoconductivity study, DRS-UV analysis and FTIR analysis.

\section{EXPERIMENT (CRYSTAL GROWTH):}

Commercially available KDP and sodium thiosulphate of GR grade having $99.9 \%$ purity were purchased, these chemicals were used for the crystal growth process. For this process, first, a saturated solution of pure KDP was prepared using distilled water which was later filtered with A1 filter paper to remove the impurities. Then, the KDP was doped with sodium thiosulphate for $1 \mathrm{~mol} \%$. The mixture solution was stirred well for about 2-4 hrs using a magnetic stirrer to get a 


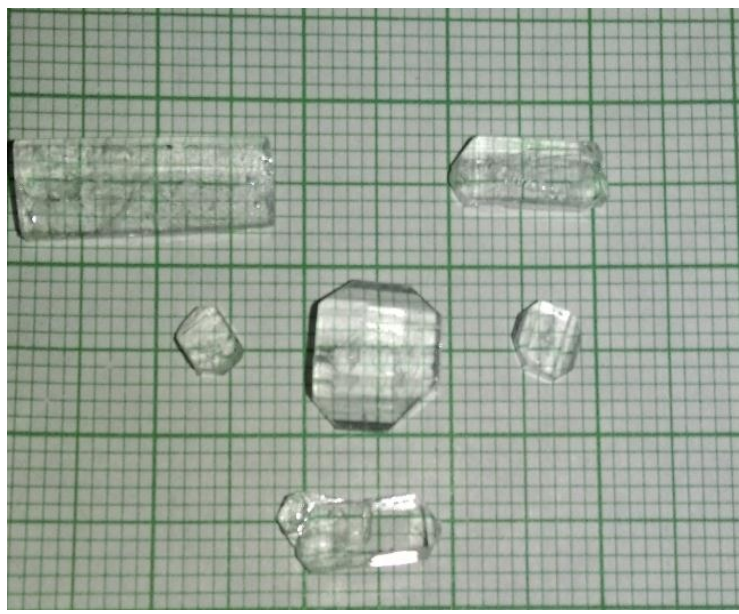

(a)Pure KDP

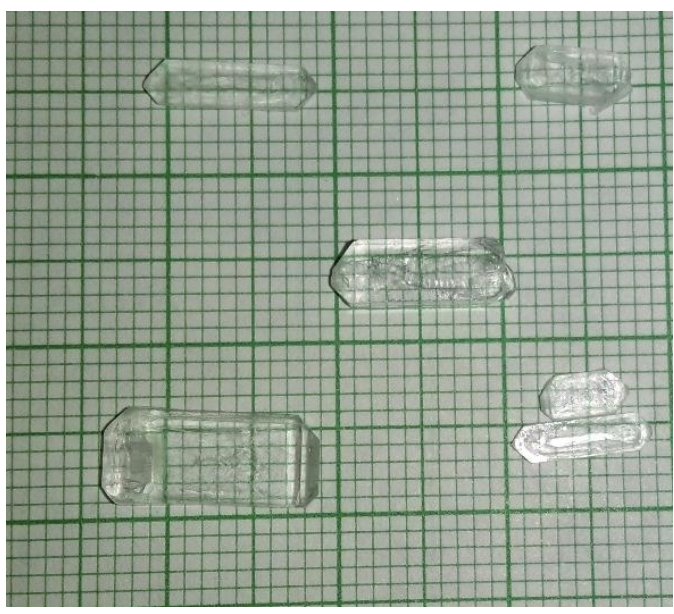

(b) Doped KDP

Figure 1: The as grown crystals of (a) Pure KDP (b) Doped KDP

homogenous solution, later the solution was filtered with A1 filter paper to remove foreign particles. The pure and doped saturated solutions were taken in two separate beakers and was covered with thin plastic paper. Holes were made on the cover for proper evaporation of the solvent and the solution was kept in a water bath for crystal growth. The bath temperature was maintained at a constant value of $35{ }^{\circ} \mathrm{C}$. After few days nucleation was found in the beakers and single crystals of good optical quality were obtained within a month. Thus the obtained crystals were non-hygroscopic, transparent and free from inclusions. The as grown pure and doped KDP crystals are shown in fig 1 .

\section{RESULTS AND DICUSSIONS:}

\section{POWDER XRD ANALYSIS:}

$\mathrm{X}$-ray powder diffraction (XRD) is one of the most powerful technique for qualitative and quantitative analysis of crystalline compounds. The technique provides information that cannot be obtained any other way. Powder XRD is useful for confirming the identity of a solid material and determining the crystallinity and phase purity. Powder X-ray diffraction analysis was carried out by using X-Ray diffractometer with $\mathrm{CuK} \alpha$ radiation $(\lambda=1.5406 \AA)$. The samples were scanned over the range $10^{\circ}-80^{\circ}$ at the rate of $2 \%$. The XRD pattern of grown sodium thiosulphate doped KDP and pure KDP is shown fig 2. The samples of pure and sodium thiosulphate doped KDP crystal were subjected for analysis in the form of finely grounded powders. The sharp and well defined Bragg peaks observed in the powder X-ray diffraction pattern confirms the crystalline nature of the compound. From the Powder XRD spectrum the entry of additives molecules into the MKP lattice can be confirmed as there is a small shift in 2 theta position and change in the intensity which is clearly shown in the spectrum. These conclude that there is a change in the internal structure of the crystal. The unit cell parameters are $\mathrm{a}=\mathrm{b}=7.453(\AA), \mathrm{c}=6.974(\AA)$, from the given data the grown crystal belongs to tetragonal class. The XRD data is verified by KDP crystal JCPDS Card no. 35- 0807 [3]

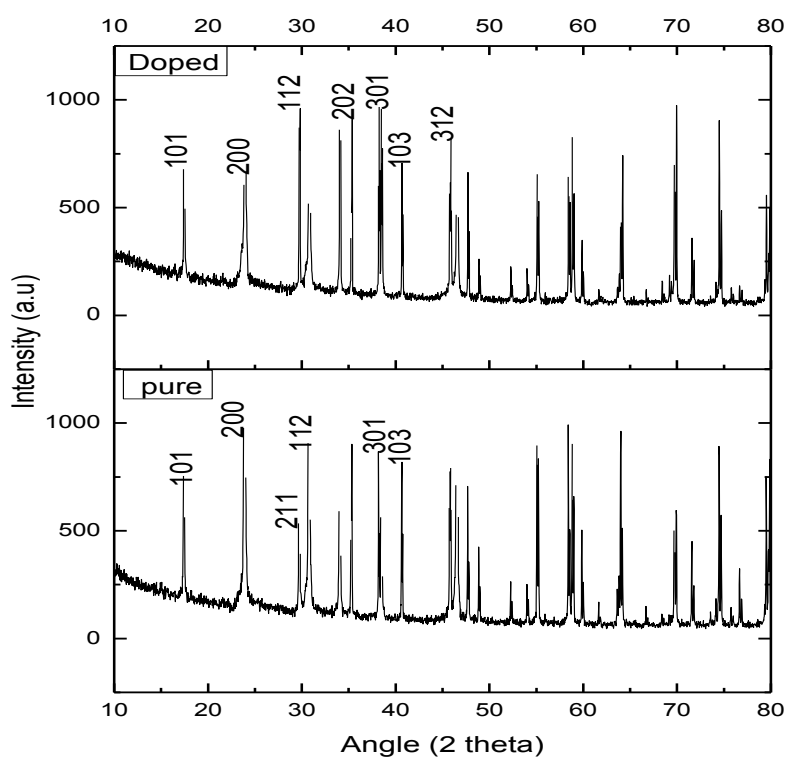

Figure 2. Powder XRD of Pure and Doped KDP crystals

\section{PHOTOCONDUCTIVITY STUDIES:}

Photoconductivity is the incremental change in the electrical conductivity of a substance upon illumination which is generated by the absorption of photons [4]. The relevant photoexcitation of freecarriers and photoconductivity are expected when the crystal is illuminated with visible or nearinfrared wavelengths. Photoconductivity studies were carried 
out for sodium thiosulphate doped KDP crystal using Keithley 485 picoammeter at room temperature, the crystal were held by copper holders. The Dark current (D) of the sample were measured by connecting a DC power supply and picometer in series. Silver paint was coated on the sample to get electrical conducts. Radiation from a halogen lamp containing iodine vapor was focused on the sample with the help of a convex lens to measure the photocurrent (P). Fig 3 shows the plot of dark current (D) and photocurrent (P) against the applied field. It was observed from the plot that both $\mathrm{D}$ and $\mathrm{P}$ of the sample increases linearly with the applied field. It is also observed that the photocurrent of doped KDP crystal was greater than the dark current, as the photocurrent is greater than the dark current it was confirmed that the crystal exhibit positive photoconductivity. This positive photoconductivity phenomenon can be attributed to generation of mobile charge carriers caused by the absorption of photons. When the energy of the incident photon, is greater than the energy gap of the crystal, free electron and hole pairs are produced in the crystal by the absorption of the incident photon i.e. when a photon hits an electron it gets mixed up to the energy of electron, thus being absorbed and these electrons and holes serves as the carriers of electric current [5]. Therefore this positive photoconductivity nature shows that the generation of mobile carriers is more in doped KDP crystal making it a worthy material for photoconductivity applications.

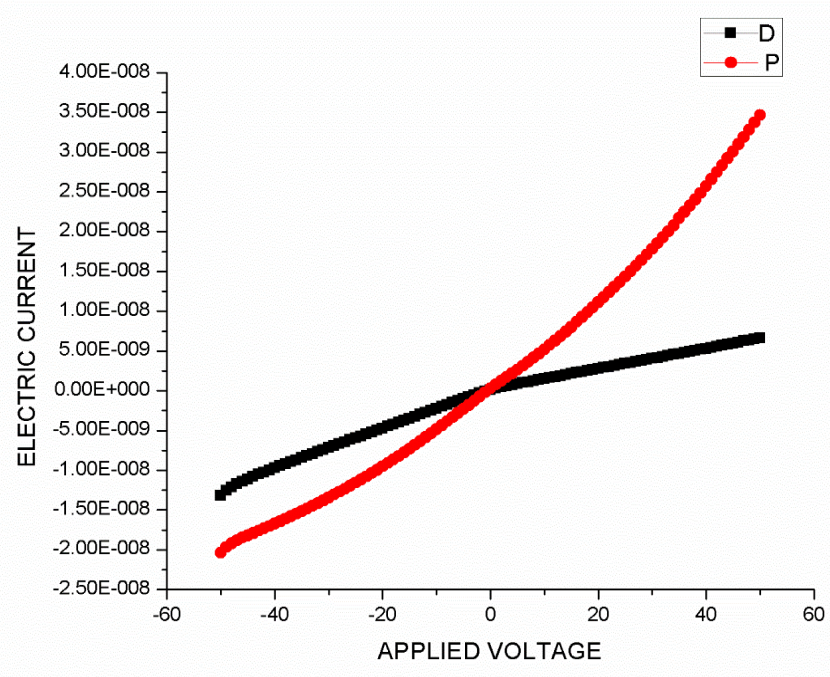

Figure 3. Photoconductivity response sodium thiosulphate doped KDP crystal

\section{DRS-UV ANALYSIS:}

The optical properties of a material are important, as they provide information on the electronic band structure, localized state and types of optical transitions. Molecules containing $\pi$ electrons or non-bonding electrons (n-electrons) can absorb the energy in the form of ultraviolet or visible light to excite electrons to higher anti-bonding molecular orbital. The more easily excited the electrons (i.e. lower energy gap between the
HOMO and the LUMO), the longer the wavelength of light it can absorb. Pure and sodium thiosulphate doped KDP crystals were finely grounded and the optical measurement was recorded in the wavelength region $200-850 \mathrm{~nm}$. From the spectrum, it was observed that both the pure and sodium thiosulphate doped KDP crystals show absorbance in the entire UV region. Absorption in the ultraviolet region arises from electronic transitions associated within the sample. It could be concluded that the sodium thiosulphate doping play a key role in improving the optical quality of KDP crystals. The addition of sodium thiosulphate seems to have increased the crystalline perfection in KDP thereby resulting in lesser absorbance when compared to pure KDP. The cut off wavelength pure crystal is around $(\sim 273 \mathrm{~nm})$ and for doped KDP crystals is around $(\sim 223 \mathrm{~nm})$ which is lower. The DRSUV data also reveals that sodium thiosulphate additives improves the optical transparency of the crystal and confirms the betterment of optical quality. Fig 4 shows the absorption percentage of the pure and doped crystals.

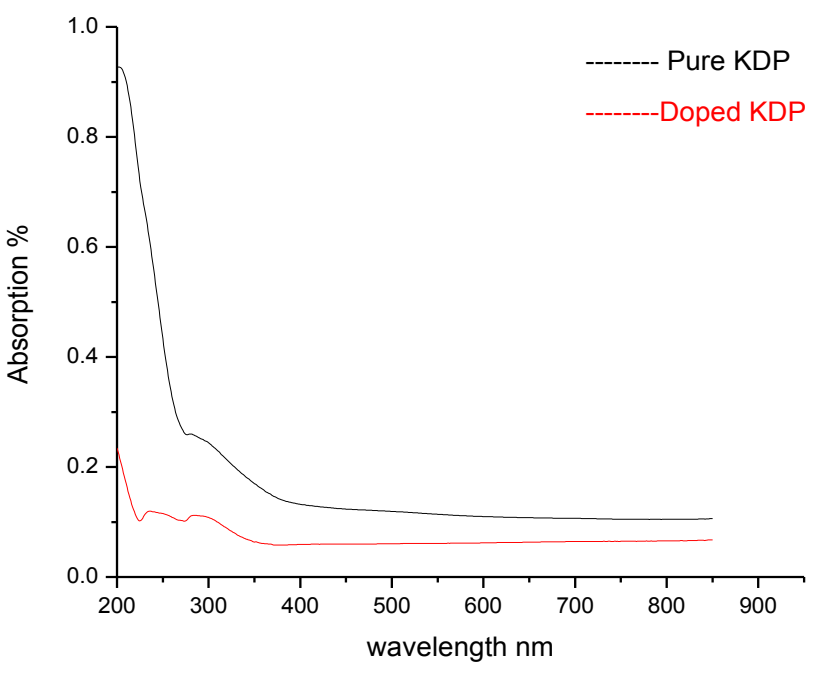

Figure 4. DRS-UV spectrum of Pure and Doped KDP crystals

\section{FT-IR ANALYSIS:}

In Fourier Transform Infrared (FT-IR) analysis the infrared radiation promotes transitions in a molecule between rotational and vibrational energy levels of the ground energy states. The FT-IRspectrumof Pure KDP and Doped KDP crystals (Figure 5) was recorded at room temperature in the spectral range $500-4000 \mathrm{~cm}^{-1}$ by ATR( Attenuated Total Reflectance) mode. The recorded spectrum reveals the presences of all functional groups occurring in KDP. The spectrum of sodium thiosulphate doped KDP crystal indicates an appreciable shift of peak positions to lower and higher values suggesting incorporation of dopants in the crystal lattice. Using the characteristic frequency values and infrared structural correlation chart, the vibrational assignments were made which is shown in table 1. 


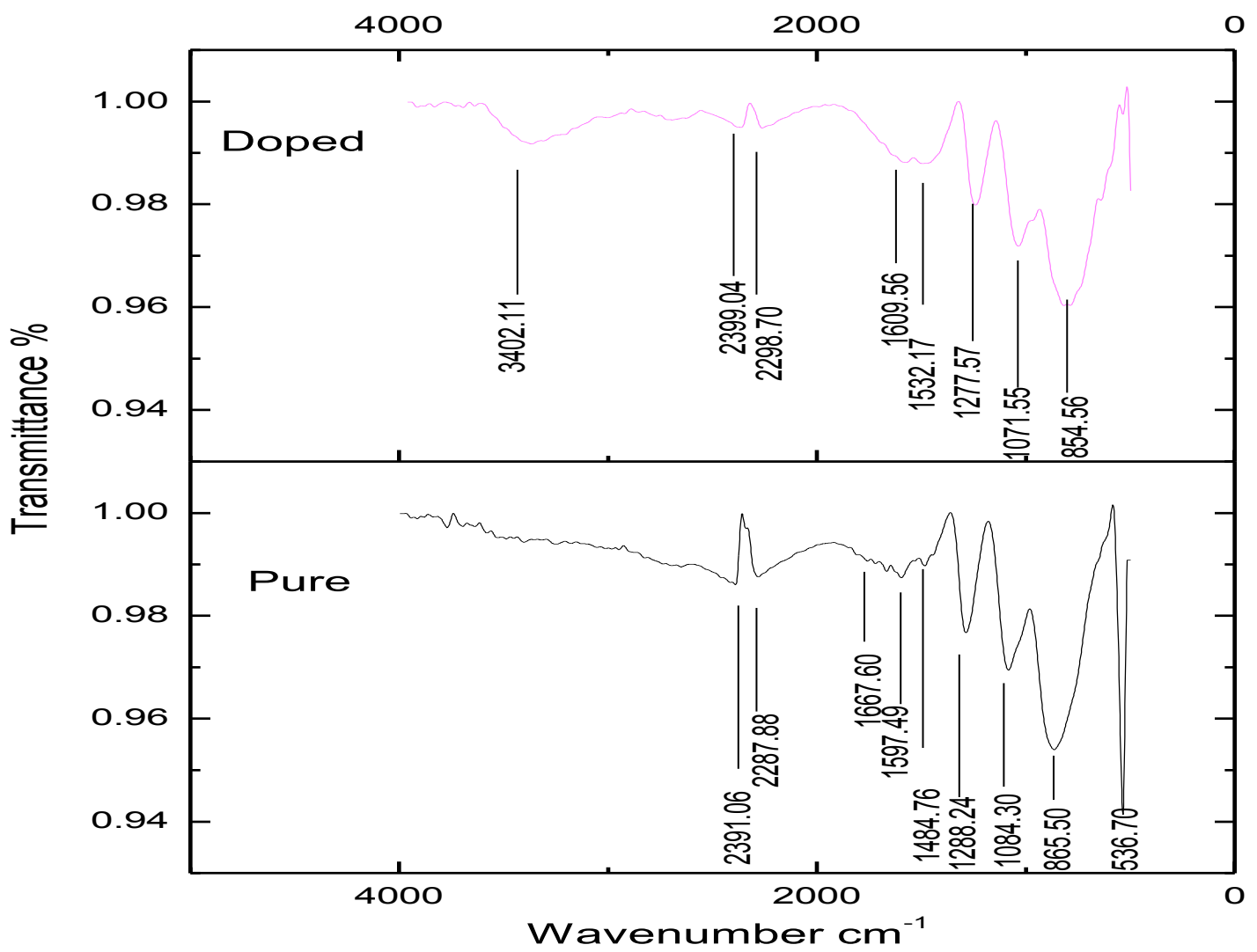

Figure 5. FT-IR spectrum of Pure and Doped KDP crystals

Table 1. FT-IR Assignments of pure and sodium thiosulfate doped KDP single crystals

\begin{tabular}{|c|c|l|}
\hline PURE KDP & SODIUM THIOSULPHATE + KDP & \multicolumn{1}{|c|}{ ASSIGNMENTS } \\
\hline- & 3402.11 & Free OH Stretching \\
\hline- & 2729.04 & P-O-H symmetric stretching \\
\hline 2391.60 & 2399.04 & P-O-H symmetric stretching \\
\hline 2282.88 & 2298.70 & P-O-H Stretching \\
\hline 1667.60 & 1609.56 & Asymmetric B-O Stretching \\
\hline 1597.49 & 1532.17 & C-O Stretching \\
\hline 1484.76 & - & O-H Stretching \\
\hline 1288.24 & 1277.57 & P-O Stretching \\
\hline 1084.30 & 1071.55 & P-O Stretching \\
\hline 865.58 & 854.56 & O-P-OH Bending \\
\hline 536.70 & - & HO-P-OH Bending \\
\hline
\end{tabular}




\section{CONCLUSION:}

Pure KDP and sodium thiosulphate doped KDP of good quality were grown by slow evaporation solution growth technique at constant temperature $\left(35^{\circ} \mathrm{C}\right)$. The grown crystals were subjected to various characterization techniques. The powder X-ray diffraction analysis confirmed the crystal structure of the pure and doped KDP from the peaks. It also gave idea about the crystal perfection. In DRS-UV absorption studies, it was revealed that the pure KDP crystal showed higher absorption wavelength, while the doped KDP crystal showed lower absorption percentage due to increase in transparency making it as a suitable material for photoconductivity application.The functional groups of the grown crystals were confirmed from FTIR analysis. The photoconductivity studies showed that the doped crystal exhibited positive photoconductivity and confirmed it as a worthy material for photoconductivity applications.

\section{ACKNOWLEDGEMENTS:}

The author gratefully acknowledge Anna University, Chennai for DRS-UV studies, IIT madras for Powder XRD, SSN college of engineering, Chennai for FT-IR and Photoconductivity studies.

\section{REFERENCES}

[1] Photoconductivity in Materials Research, Monica Brinza, Jan Willekens Mohammed Benkhedir Guy Adriaenssens

[2] Nihal U. Obeyesekere, 9 - Pitting corrosion, Editor(s): A.M. El-Sherik,In Woodhead Publishing Series in Energy, Trends in Oil and Gas Corrosion Research and Technologies, Woodhead Publishing, 2017, Pages 215248, JCPDS Card no. 35- 0807

[3] JCPDS Card no. 35- 0807

[4] S. Follonier, M. Fierz, I. Biaggio, U. Meier, C. Bosshard, and P. G"unter, "Structural, optical, and electrical properties of the organic molecular crystal 4N,N-dimethylamino-4'-N'-methyl stilbazolium tosylate," Journal of the Optical Society of America B: Optical Physics, vol. 19, no. 9, pp. 1990-1998, 2002

[5] B. Babu, J. Chandrasekaran, S. Balaprabhakaran, P. Ilay abarathi "Optical, structural and electrical properties of pure and urea doped KDP crystals", Materials SciencePoland, January 2013, Volume 31, Issue 1, pp 151-157

[6] 